\title{
Criminologie
}

\section{Les réseaux de coauteurs : 40 ans de collaborations à la revue Criminologie}

\author{
Co-authorship networks: 40 years of collaborations in the \\ journal Criminologie
}

\section{Las redes de coautores: 40 años de colaboraciones en la revista Criminologie}

\section{David Décary-Hétu et Sabrina Vidal}

Volume 51, numéro 1, printemps 2018

50 ans de Criminologie

URI : https://id.erudit.org/iderudit/1045308ar

DOI : https://doi.org/10.7202/1045308ar

\section{Aller au sommaire du numéro}

Éditeur(s)

Les Presses de l’Université de Montréal

ISSN

0316-0041 (imprimé)

1492-1367 (numérique)

Découvrir la revue

Citer cet article

Décary-Hétu, D. \& Vidal, S. (2018). Les réseaux de coauteurs : 40 ans de collaborations à la revue Criminologie. Criminologie, 51(1), 55-78.

https://doi.org/10.7202/1045308ar

\section{Résumé de l'article}

La recherche scientifique cherche la plupart du temps à comprendre la nature mais se tourne aussi à l'occasion vers elle-même pour mieux comprendre la genèse des connaissances scientifiques. Cette quête s'est traduite par une littérature importante portant sur les réseaux de collaborations entre auteurs pour produire des textes scientifiques. L'objectif général de cette branche de recherche est d'expliquer la structure des collaborations et de déterminer les corollaires de la performance dans le monde universitaire. Cet article vise à combler un manque de connaissance sur les réseaux de collaboration francophones en étudiant 40 ans de collaborations entre auteurs de la revue Criminologie. La méthodologie adopte une approche égocentrique et sociométrique et caractérise les liens entre les coauteurs de la revue. Nos résultats démontrent une augmentation des collaborations dans le temps, mais sans nécessairement la création d'une seule et unique communauté d'auteurs qui contrôlerait la diffusion des connaissances dans la revue. Les acteurs clés sont présentés mais ne montrent aucune stabilité dans le temps. 


\title{
Les réseaux de coauteurs: 40 ans de collaborations à la revue Criminologie
}

\author{
David Décary-Hétu ${ }^{1}$ \\ Professeur adjoint \\ École de criminologie, Université de Montréal \\ david.decary-hetu@umontreal.ca \\ Sabrina Vidal \\ Candidate au doctorat \\ École de criminologie, Université de Montréal \\ sabrina.vidal@umontreal.ca
}

RÉSUMÉ • La recherche scientifique cherche la plupart du temps à comprendre la nature mais se tourne aussi à l'occasion vers elle-même pour mieux comprendre la genèse des connaissances scientifiques. Cette quête s'est traduite par une littérature importante portant sur les réseaux de collaborations entre auteurs pour produire des textes scientifiques. L'objectif général de cette branche de recherche est d'expliquer la structure des collaborations et de déterminer les corollaires de la performance dans le monde universitaire. Cet article vise à combler un manque de connaissance sur les réseaux de collaboration francophones en étudiant 40 ans de collaborations entre auteurs de la revue Criminologie. La méthodologie adopte une approche égocentrique et sociométrique et caractérise les liens entre les coauteurs de la revue. Nos résultats démontrent une augmentation des collaborations dans le temps, mais sans nécessairement la création d'une seule et unique communauté d'auteurs qui contrôlerait la diffusion des connaissances dans la revue. Les acteurs clés sont présentés mais ne montrent aucune stabilité dans le temps.

MOTS CLÉS - Réseaux de coauteurs, analyse de réseaux, études longitudinales, réseaux francophones.

\section{Introduction}

L'étude de la construction des savoirs scientifiques cherche à comprendre l'évolution des sciences. Cette construction s'actualise, selon certains (Popper, 2014), à l'aide d'expérimentations qui tentent de

1. École de criminologie, Université de Montréal, Pavillon Lionel-Groulx, C. P. 6128, succ. Centre-ville, Montréal (Québec), Canada, H3C 3J7.

Criminologie, vol. 51, nº 1 (2018) 
falsifier les théories faibles une par une jusqu'à ce qu'il n'en reste que des vraies. L'objectif de la science serait ainsi d'éliminer les faux savoirs. D’autres (Kuhn, 1981) conçoivent plutôt l'évolution de la science sur le modèle politique avec des institutions, mais aussi, et surtout, des révolutions. Chaque science posséderait son paradigme, une vérité générale qui guide autant les questions que les chercheurs peuvent se poser que les méthodes à utiliser pour y répondre. Périodiquement, ce paradigme serait mis à l'épreuve et ne pourrait apporter des solutions aux questions posées. Cet échec amènerait une phase de remise en question qui, à terme, mènerait à l'émergence d'un nouveau paradigme qui répondrait mieux aux observations dans la nature.

Des chercheurs comme Popper et Kuhn adoptent une vision très générale des sciences et de la construction des savoirs scientifiques. Ces savoirs peuvent paraittre abstraits, mais sont dans les faits le fruit du travail et des collaborations entre chercheurs. Un champ de recherche s'est développé au cours des dernières décennies afin d'étudier cette question de la construction des savoirs scientifiques, mais sur un plan microscopique et surtout humain. Ces recherches ont défini les collaborations comme étant un mécanisme clé pour l'avancement des connaissances et surtout comme une mesure de production des connaissances qui aurait de plus en plus d'utilité (Melin et Persson, 1996). L'étude des collaborations permet d'illustrer à la fois la migration et la propagation des savoirs scientifiques (Largeron-Leteno et Massard, 2001). Les chercheurs seraient des êtres sociaux qui discutent et échangent leurs idées. Pour comprendre ces échanges, des études se sont intéressées à l'intensité des collaborations et aux mécanismes de communication (Glänzel, 2002; Uddin, Hossain, Abbasi et Rasmussen, 2012; Velden, Haque et Lagoze, 2010).

Cette recherche bâtie sur ces recherches passées cherche à étendre notre compréhension de la construction des savoirs en étudiant un réseau de collaboration entre des auteurs d'une revue scientifique, la revue Criminologie, sur la période des 40 dernières années. Elle innove en transposant un cadre d'analyse déjà connu à une science humaine et à une langue, le français, rarement étudiées dans la littérature scientifique. La première section de l'article présente l'évolution des réseaux de coauteurs au cours des dernières décennies. Nous verrons que ces réseaux se sont densifiés à travers le temps et que les auteurs les plus performants ont misé sur les collaborations internationales et interinstitutions. Nous présenterons par la suite nos données, extraites directe- 
ment du site web de l'éditeur de la revue. Des analyses tant des réseaux locaux des auteurs que du réseau global des auteurs seront présentées. Nos résultats nous permettent de décrire l'évolution des liens entre les auteurs de Criminologie et de rejoindre en partie les résultats des études passées. La nature thématique de la revue assure cependant une certaine stabilité dans la structure des liens entre les auteurs.

\section{Les réseaux de coauteurs}

Un réseau de collaborateurs scientifiques se forme lorsque deux ou plusieurs chercheurs travaillent ensemble pour créer du savoir scientifique, menant généralement à une communication scientifique orale ou écrite (Katz et Martin, 1997). Plusieurs raisons peuvent expliquer le désir de collaborer chez les chercheurs (Katz et Martin, 1997; Laudel, 2002). Les collaborations permettent tout d'abord de répartir les coûts des recherches, autant sur le plan financier qu'humain (Wuchty, Jones et Uzzi, 2007). Les recherches sont en effet de plus en plus complexes et lourdes et aucun chercheur ne peut maîtriser toutes les techniques et théories nécessaires à la réalisation d'études empiriques complexes. Les coûts des recherches ont suivi une courbe similaire aux besoins humains et la répartition des coûts entre plusieurs projets financés peut augmenter les chances de réalisation d'un projet. Les problèmes étudiés sont par ailleurs de plus en plus complexes et une approche multidisciplinaire peut amener les chercheurs à remettre en question leurs paradigmes et à trouver des réponses plus complètes, fidèles et valides. Finalement, d'un point de vue politique, les collaborations permettent de faire rayonner les institutions et les nations, et maximisent donc l'impact des investissements.

Toutes les collaborations ne mènent pas nécessairement à la publication de communications. Malgré cela, l'étalon pour mesurer la collaboration scientifique est la publication, même si cette mesure est imparfaite (Katz et Martin, 1997; Paul-Hus, Mongeon, Sainte-Marie et Larivière, 2016). D’autres données, comme les remerciements, permettent de pallier quelque peu les limites des études sur les coauteurs, mais cette mesure n'est pas une panacée en soi non plus (Paul-Hus et al., 2016). Cette mesure est cependant rarement intégrée aux études, possiblement pour des questions techniques de collecte et d'analyse de ces données qui sont moins structurées que les listes d'auteurs. Larivière et al. (2016) expliquent par ailleurs que tous les collaborateurs n'ont pas 
une chance égale de devenir auteur d'une publication. Le fait d'avoir plus d'expérience et de participer aux tâches centrales de production de connaissances (ex.: conception de l'étude, conception théorique) augmente les chances de devenir auteur, comparé à des tâches plus simples comme la réalisation des manipulations techniques.

Un réseau de coauteurs prend de l'expansion au fur et à mesure que ses participants collaborent entre eux et avec d'autres auteurs, créant ainsi des liens directs et indirects entre les différents acteurs ( $\mathrm{Li}$, Liao et Yen, 2013; Yin, Kretschmer, Hanneman et Liu, 2006). Les réseaux de coauteurs ont grandement augmenté dans les années 1990 (Henriksen, 2016) avec l'arrivée massive des technologies de communication et la baisse des coûts de transport (Henriksen, 2016; Katz et Martin, 1997). En parallèle, l'étude des réseaux de coauteurs s'est énormément développée dans les années 2000 avec la création de larges banques de données de textes scientifiques accessibles directement sur Internet. Les objectifs spécifiques visés par ces études sont variés, mais tournent généralement autour de la caractérisation des liens entre auteurs et de la détection d'acteurs clés dans des réseaux de coauteurs. L'identification des auteurs clés permet d'expliquer la distribution de la réussite, réussite définie généralement par le nombre d'articles publiés et le nombre de citations reçues (Abbasi, Altmann et Hossain, 2011). Elle permet aussi de connaître les meilleurs chercheurs avec qui travailler en vue par exemple de développer des programmes d'intervention (Morel, Serruya, Penna et Guimarães, 2009; Yu, Shao et Duan, 2011).

Pavlov et Ichise (2007) ont développé un algorithme d'apprentissage supervisé par les machines pour identifier les acteurs clés avec un taux de réussite très élevé. Cette approche ancrée dans le génie informatique est plutôt l'exception, la quasi-totalité des chercheurs impliqués dans l'étude des réseaux de coauteurs optant plutôt pour des outils d'analyse de réseaux sociaux plus traditionnels. L'analyse de réseaux sociaux est définie comme l'analyse de liens entre des acteurs, ce qui permet de caractériser non seulement la structure d'un réseau, mais aussi la position des acteurs qui le composent. Deux approches différentes permettent de comprendre les réseaux sociaux : l'approche égocentrique et l'approche sociométrique. L'approche égocentrique sert à analyser le réseau local de chaque acteur et permet de comprendre comment les auteurs construisent leur réseau personnel de collaboration (Chung et Hossain, 2009). La densité des liens dans le réseau local, sa capacité à transmettre l'information efficacement et la présence de liens forts 
(collaborations répétées) font partie des mesures utilisées pour caractériser les réseaux locaux de coauteurs (Abassi et al., 2011).

L'approche sociométrique est beaucoup plus utilisée que l'approche égocentrique et étudie les réseaux de coauteurs en entier plutôt que le réseau local de chaque acteur. Selon Newman (2004), l'analyse sociométrique des réseaux de coauteurs permet de mieux comprendre la société universitaire en mettant de l'avant la structure de la genèse des connaissances. De très nombreuses mesures ont été utilisées pour caractériser la structure des réseaux de coauteurs et identifier leurs acteurs clés. Ces mesures peuvent être divisées en quatre grandes familles: 1) l'étude des liens directs entre auteurs à l'aide de mesures comme le degré de centralité; 2) l'étude des liens indirects entre auteurs, soit la capacité des auteurs à contrôler les collaborations dans un domaine ou à une certaine époque, à l'aide de mesures comme le degré d'intermédiarité; 3) l'étude de la proximité des auteurs, soit la distance entre les auteurs d'un même réseau, à l'aide de mesures comme la centralité de proximité; et 4) l'étude de la formation de cliques, soit des sous-groupes d'acteurs qui collaborent étroitement entre eux, à l'aide de mesures comme la densité et l'analyse de factions.

Les données permettant de bâtir des réseaux d'auteurs proviennent de plusieurs sources. Elles peuvent tout d'abord être extraites directement des articles scientifiques eux-mêmes en repérant, pour chacun, les lignes contenant les noms des auteurs (Abbasi et al., 2012). Les données peuvent aussi être extraites des sites web des revues en y effectuant des recherches à l'aide des noms d'auteurs (De Stefano, Fuccella, Vitale et Zaccarin, 2013). L'étendue des données collectées varie d'une seule revue pour les recherches les plus simples (Lu et Feng, 2009) à une vingtaine de revues (Velden et al., 2010), voire plus. Lutilisation de plusieurs revues permet d'augmenter le nombre d'auteurs et de liens dans le réseau et ainsi d'étudier un plus grand réseau de collaborateurs. Certaines recherches ciblent plusieurs domaines de recherches dans leurs études afin d'établir les collaborations entre les domaines ou encore de comparer les réseaux des différents domaines de recherches entre eux. Par exemple, la recherche de Newman (2004) compare les réseaux des chercheurs en biologie, en physique et en mathématique afin de voir quel domaine collabore plus que les autres. Les résultats indiquent que les chercheurs en biologie sont ceux qui collaborent le plus, ce qui serait expliqué par les nombreuses expérimentations que nécessitent les recherches en biologie (Newman, 2004). Plusieurs 
auteurs cherchent aussi à caractériser les réseaux plus locaux comme ceux des chercheurs chinois (Yu et al., 2011), turcs (Gossart et Özman, 2009) ou brésiliens (Morel et al., 2009).

Les résultats passés montrent que les réseaux de coauteurs se sont densifiés au cours des dernières décennies (Börner, Dall'Asta, Ke et Vespignani, 2005). La distance entre les auteurs deviendrait donc de plus en plus petite (Barabâsi et al., 2008). La proportion d'articles publiés par un seul auteur aurait drastiquement diminué, passant de $25-30 \%$ de tous les articles publiés dans un domaine à $10 \%$ au cours des dernières décennies (Glänzel et Schubert, 2004; Uddin et al., 2011). De ces articles, $30 \%$ auraient 2 auteurs et $25 \%$ auraient 3 auteurs (Liu, Bollen, Nelson et Van De Sompel, 2005). Les auteurs avaient en 1980 une moyenne de 2,6 coauteurs dans leur réseau égocentrique; ils étaient en 2000 plus de 50\% plus élevés, à 4,16 (Glänzel et Schubert, 2004). Ces hausses seraient présentes dans la plupart des domaines (Acedo, Barroso, Casanueva et Galán, 2006; Henriksen, 2016). Les études en sciences de la vie et en sciences pures auraient des réseaux de collaboration plus importants que les autres domaines en se basant sur les liens de coauteurs, mais ces différences tendent à s'estomper lorsque les chercheurs tiennent compte des autres aspects des collaborations comme les remerciements et les tâches de collecte de données (Paul-Hus et al., 2016).

Ces augmentations de proportion d'articles avec plusieurs auteurs seraient dues en partie à l'augmentation des collaborations internationales (Glänzel et Schubert, 2004) et des collaborations entre les institutions (Liu et al., 2005; Morel et al., 2009). Lorsqu'une bonne relation de collaboration s'installe entre deux auteurs, il arrive également souvent que les auteurs répètent l'expérience de produire un article ensemble ( $\mathrm{Li}$ et al., 2013). Tous les chercheurs n'auraient pas été en mesure de bonifier leur réseau de coauteurs (Glänzel et Schubert, 2004), créant ainsi une division entre des auteurs performants et d'autres qui le sont moins. Les auteurs aux nombreux coauteurs seraient en mesure d'écrire plus d'articles, de rayonner davantage et d'avoir accès à plus de sources de financement (Lee et Bozeman, 2005). Ils auraient aussi accès à de nouvelles techniques et technologies qui élèvent la qualité et la quantité des résultats obtenus, et ce, dans la plupart des disciplines de recherche (Babchuk, Keith et Peters, 1999; Glänzel et Schubert, 2004; Kronegger, Ferligoj et Doreian, 2011). Dans ce cas, il ne serait pas essentiel de publier avec des auteurs performants pour 
exceller (Abbasi, Altmann et Hossain, 2011), par contre, les liens faibles avec des auteurs impliqués dans plusieurs réseaux de coauteurs seraient un atout.

Les méthodologies pour déterminer les chercheurs plus performants sont très variées dans les recherches passées. Liu et al. (2005) proposent une nouvelle mesure, l'AuthorRank, basée sur l'algorithme de PageRank qui propulse le moteur de recherche Google. Cet algorithme donne du poids aux acteurs qui sont régulièrement référencés par leurs pairs. Selon Liu et al. (2005), l'AutborRank donnerait de meilleurs résultats que les mesures basées sur les liens directs, les liens indirects et la proximité. Pour plusieurs autres chercheurs, ces mesures couramment utilisées en analyse de réseaux resteraient très pertinentes. Badar, Hite et Badir (2013) trouvent que les liens directs et la proximité sont corrélés aux mesures de performances comme le nombre de citations, mais que les liens indirects ne joueraient pas de rôle décisif. Yan et Ding (2009) confirment l'importance des liens directs. Li et al. (2013) trouvent plutôt l'inverse et mettent l'accent sur les liens indirects. Leur modèle qui inclut aussi de nouveaux concepts comme l'exploration d'équipe démontre les relations complexes qui peuvent survenir entre la structure d'un réseau de coauteurs et la performance.

Il n'existerait pas un seul acteur central dans les réseaux de coauteurs (Cheong et Corbitt, 2009; Erfanmanesh, Rohani et Abrizah, 2012). Les réseaux dans chaque domaine seraient vastes et compétitifs et compteraient beaucoup d'auteurs reconnus. Certaines populations d'auteurs seraient plus à même de se classer parmi les auteurs clés, comme ceux provenant des États-Unis. Les auteurs de régions moins favorisées comme la Turquie éprouveraient plus de difficulté à cimenter des collaborations internationales (Gossart et Özman, 2009). La structure des réseaux de coauteurs d'un chercheur aurait donc un impact sur sa performance en matière de quantité et de qualité de publications. Une position centrale au sein du réseau serait avantageuse en ce qui concerne le partage de connaissances et le nombre de citations dans d'autres publications. L'étude des réseaux de coauteurs permet de cibler les auteurs les plus prolifiques au sein de domaines de recherches et d'identifier les collaborateurs clés qui permettent d'augmenter le nombre et l'impact de chaque publication. 


\section{Vers une nouvelle caractérisation des réseaux de coauteurs}

L'analyse des réseaux de coauteurs donne une image imparfaite des collaborations scientifiques (Katz et Martin, 1997; Paul-Hus et al., 2016), mais représente à ce jour le meilleur outil pour comprendre comment la science est produite ainsi que le contexte de cette production. La production du savoir à plusieurs, l'intensité des liens entre les coauteurs et la répétition des liens entre les auteurs sont autant d'indicateurs et de prédicteurs de la fécondité et de la quantité des publications produites. Les réseaux de coauteurs permettent par ailleurs de mieux comprendre les jeux de pouvoir entre auteurs dans un domaine et ainsi de relever le contrôle potentiel de certains auteurs sur des publications ou des médias de communication.

Les recherches passées se sont particulièrement intéressées au monde anglo-saxon ainsi qu'aux revues en sciences dites dures (sciences de la vie, pures et de la nature). L'anglais étant la langue de la science (et des communications scientifiques dans bien des cas), il n'est guère surprenant de constater que les recherches dans la langue de Shakespeare dominent la littérature sur les analyses de réseaux de coauteurs. Par leur dominance, il est fort probable que ces réseaux soient plus étendus et plus denses que les réseaux de coauteurs issus de minorités linguistiques ou encore de domaines de recherche moins dynamiques et compétitifs. Les auteurs dans d'autres domaines ont en effet probablement une pression plus modeste de performer et n'ont donc pas la même motivation à créer de grands groupes d'auteurs qui pourront leur être utiles dans le futur.

La célébration, cette année, du 50e anniversaire de la revue Criminologie nous offre une opportunité intéressante d'appliquer les connaissances développées jusqu'ici en analyse de réseaux de coauteurs à une communauté plus limitée, soit celle des auteurs de Criminologie. La revue Criminologie est l'une des deux revues francophones à s'intéresser au phénomène criminel avec la Revue canadienne de criminologie et de justice pénale. Deux autres revues francophones à l'international s'intéressent spécifiquement au phénomène criminel, soit Déviance et société et la Revue internationale de criminologie et de police technique et scientifique. L'impact de la revue Criminologie mesuré par son index h5 est similaire ou supérieur aux autres revues francophones selon les statistiques de Google Scholar. À la différence des autres revues, Criminologie est cependant une revue thématique et est publiée deux fois par année. 
Cette revue très importante pour le développement de la criminologie dans la francophonie attire un bassin d'auteurs très large, qui inclut autant des chercheurs professionnels que des étudiants et des praticiens. La diversité de ses auteurs, conjuguée à sa langue de publication (le français), pourrait avoir un impact important sur la structure des liens entre les auteurs. Pour cette raison, l'objectif général de cet article est de caractériser l'évolution des réseaux de coauteurs de la revue Criminologie au cours des 40 dernières années. Les résultats de cette étude nous permettront d'étudier les collaborations scientifiques et d'alimenter les futures recherches qui s'intéressent aux structures derrière les collaborations scientifiques.

\section{Données}

Les collaborations entre auteurs sont généralement opérationnalisées à travers l'étude des liens entre auteurs d'un même article. Étant donné la nature thématique de la revue Criminologie, il aurait aussi été possible d'étudier les liens entre les auteurs d'un même numéro. Afin de pouvoir mettre en contexte nos résultats, nous nous sommes cependant concentrés sur les liens entre les auteurs d'un même article afin de pouvoir comparer nos résultats aux études passées.

Les données pour cette recherche ont été collectées directement sur le site web d'Érudit, qui publie la version numérique de la revue Criminologie. Le site héberge une page unique pour chaque article de la revue et cette page contient le titre de l'article, son année de publication et une liste des noms des auteurs. Les auteurs de cet article ont développé un logiciel spécialisé qui a indexé les URL de toutes les pages des articles de la revue Criminologie, puis les a téléchargées. Au total, 717 pages d'articles, allant de 1977 à 2016 inclusivement, ont été téléchargées. Ce même logiciel a par la suite extrait, pour chaque article, les noms des auteurs et a créé quatre matrices de relation non dirigées dichotomiques. Ces matrices représentent tous les liens des coauteurs pour quatre périodes temporelles, soit de 1977 à 1986, de 1987 à 1996, de 1997 à 2006 et de 2007 à 2016. La délimitation des périodes a d'abord été réalisée pour des raisons pratiques, 2016 étant la dernière année complète pour laquelle des données étaient disponibles. La décision de diviser les années antérieures en périodes de 10 ans permet de mieux comparer l'évolution des réseaux de coauteurs, un objectif important des recherches passées, et de limiter le nombre de données à présenter 
pour le lecteur. Elle permet également d'avoir un nombre relativement important d'auteurs dans chaque réseau, la revue ne publiant que deux numéros par année.

\section{Méthodes}

Pour caractériser l'évolution des réseaux de coauteurs de la revue Criminologie au cours des 40 dernières années, nous avons analysé les matrices de relation à l'aide du logiciel UCINET (Borgatti, Everett et Freeman, 2002), un logiciel couramment utilisé pour réaliser des analyses de réseaux.

Nos analyses commencent par une inspection visuelle de la forme et de la structure des liens dans les matrices. Chaque acteur est représenté par un point dont la grosseur varie en fonction du nombre de liens avec des coauteurs. L'épaisseur des liens varie en fonction de l'intensité de la relation entre deux auteurs. Le reste de nos analyses se déroule en cinq temps.

Premièrement, des analyses descriptives présentent les réseaux de coauteurs pour chaque décennie. Ces analyses nous permettent de discuter de l'évolution dans le temps du nombre d'articles publiés dans la revue Criminologie, du nombre d'auteurs, de la proportion d'auteurs publiant seuls, du nombre d'auteurs moyen par article, du nombre de liens de coauteurs et du nombre moyen de liens par auteurs. Les résultats de ces analyses descriptives dressent un portrait sommaire de l'état des collaborations ayant mené à au moins une publication dans la revue Criminologie.

La deuxième série d'analyses présente la structure des réseaux de coauteurs. Cette structure est caractérisée à travers quatre mesures tirées d'une approche sociométrique d'analyse de réseau, soit la densité des liens, le coefficient d'agglomération, le degré de centralisation et la centralité d'information. La densité mesure le ratio entre le nombre de liens théoriquement possibles dans un réseau et le nombre de liens observés. Un coefficient de densité de 1 signifie que tous les acteurs sont connectés à tous les autres dans un réseau. Ce type de réseau est très redondant et suppose une forte proximité entre les acteurs d'un réseau. Le coefficient d'agglomération vise à détecter la tendance d'un réseau à former des cliques, des sous-groupes d'acteurs ayant une forte densité de liens entre eux. Un réseau ayant un coefficient d'agglomération plus élevé que sa densité aura tendance à former de petites cliques et à limiter la diffusion 
d'informations et de ressources au réseau en général. La centralisation de degré mesure la tendance d'un réseau à concentrer tous ses liens vers un seul acteur. Un coefficient de 1 indique donc que tous les liens d'un réseau sont dirigés vers ou depuis un seul acteur. Cet acteur a un pouvoir direct sur les autres acteurs et peut influencer leurs actions. Finalement, la centralité d'information permet de mesurer la distance qui sépare les acteurs d'un réseau. Un coefficient de 1 indique que les acteurs peuvent rapidement rejoindre les autres acteurs du réseau et qu'il existe ainsi une grande collaboration entre les auteurs. La centralité d'information mesure aussi une forme de pouvoir et donc d'influence sur les acteurs, mais à travers des acteurs directs et indirects plutôt que simplement directs comme dans le cas du degré de centralisation.

La troisième série d'analyses raffine les analyses précédentes sur la formation de cliques dans le réseau, un concept important dans l'étude des collaborations scientifiques. Elle est actualisée à travers un recensement des triades. Ce recensement sélectionne, à tour de rôle, tous les trios d'acteurs possibles dans chacune des matrices. Il comptabilise ensuite le nombre de triades comportant 0, 1, 2 et 3 liens. La proportion de chaque type de triade est indicative de la tendance du réseau à former des cliques (forte proportion de triades à 3 liens) ou son inverse (forte proportion de triades à 0 lien). L'évolution de la proportion de chaque type de triade est aussi importante pour comprendre les tendances dans les collaborations entre auteurs.

La quatrième série d'analyses adopte une approche égocentrique et cherche à comprendre la taille des réseaux de collaborations de chaque auteur ayant publié dans la revue Criminologie ainsi que la densité des liens entre les collaborateurs de chaque auteur. Un auteur qui collabore avec le même groupe d'auteurs aura tendance à avoir une densité égocentrique très élevée.

La dernière série d'analyses est de nature descriptive et identifie les acteurs qui forment le cœur de chacune des matrices de relation. Cette analyse cherche à savoir si des acteurs formant une clique sont centraux par leur positionnement dans leur réseau. Le nom des acteurs n'est ici important que pour des raisons historiques, mais nous permettra tout de même de vérifier si certains auteurs font partie du cœur du réseau dans plus d'une décennie. Cela nous permettra de mieux comprendre la stabilité dans les auteurs clés de la revue Criminologie. 


\section{Résultats}

La visualisation des liens nous offre un premier point de repère pour caractériser plus qualitativement l'évolution des réseaux de coauteurs de la revue Criminologie au cours des 40 dernières années. La Figure 1 présente les quatre matrices de collaborations entre les auteurs de la revue Criminologie, et ce, pour chaque période temporelle.

\section{F I G URE 1}

Sociogrammes des liens de coauteurs

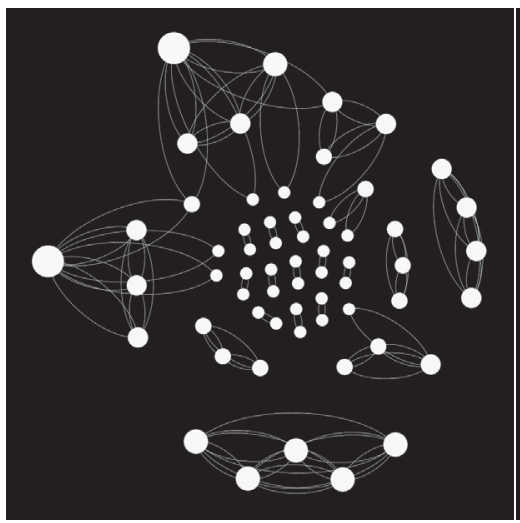

1977-1986

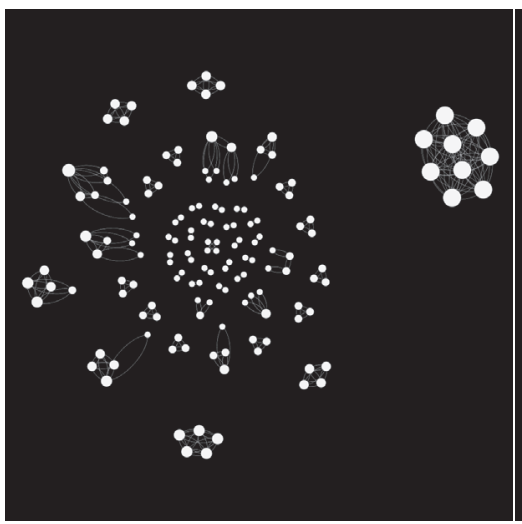

1997-2006

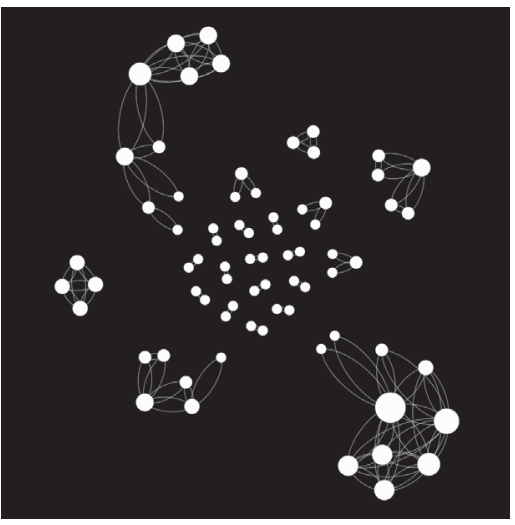

1987-1996

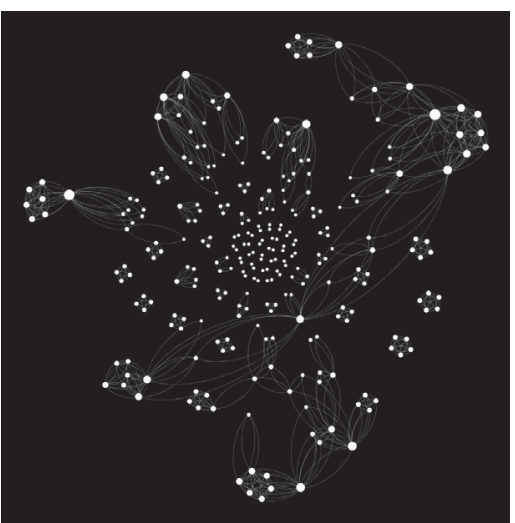

2007-2016

La Figure 1 montre que le nombre d'auteurs a progressivement augmenté à travers les quatre décennies étudiées avec un nombre d'auteurs beaucoup plus important pour les années 2007-2016. La taille des 
acteurs apparaît beaucoup plus homogène dans le cadran en haut à gauche qu'en bas à droite, semblant indiquer une distribution moins équitable des liens plus les années avancent. En 2007-2016, les acteurs sont en effet généralement de taille très petite, avec quelques acteurs qui ressortent du lot seulement. La Figure 1 indique aussi une diminution de la distance entre les acteurs. La présence de cliques reste importante, mais ces cliques sont plus connectées les unes aux autres pour former de larges composantes, particulièrement pour les années 2007-2016. Des groupes d'auteurs semblent donc travailler ensemble, mais un ou plusieurs d'entre eux cherchent aussi à collaborer avec d'autres à l'extérieur de leur clique immédiate.

TABLEA U 1

Statistiques descriptives des réseaux de coauteurs

\begin{tabular}{|l|r|r|r|r|}
\hline & $1977-1986$ & $1987-1996$ & $1997-2006$ & $2007-2016$ \\
\hline Nombre d'articles & 159 & 152 & 163 & 243 \\
\hline Nombre d'auteurs & 120 & 145 & 207 & 354 \\
\hline $\begin{array}{l}\text { Nombre d'auteurs publiant } \\
\text { seuls (proportion) }\end{array}$ & $59(49 \%)$ & $72(50 \%)$ & $58(28 \%)$ & $69(19 \%)$ \\
\hline Nombre moyen d'auteurs par article & 1,3 & 1,4 & 1,7 & 1,9 \\
\hline Nombre de liens de coauteurs & 62 & 79 & 167 & 427 \\
\hline Nombre moyen de liens par auteur & 0,5 & 0,5 & 0,8 & 1,2 \\
\hline
\end{tabular}

Le nombre d'articles indexés par notre logiciel spécialisé est relativement stable pour les trois premières décennies $(\min =152 ; \max =163)$, mais augmente de manière importante au cours des 10 dernières années avec 243 articles. Cela laisse supposer une augmentation de la taille des numéros de la revue. Le nombre d'auteurs contribuant à la revue est lui aussi en augmentation, passant de 120 en 1977-1986 à 354 en 2007-2016 (+295\%). Pendant les 20 premières années, la moitié de ces auteurs contribuaient seuls à la revue. Ces 20 dernières années, cette proportion a cependant chuté de manière importante pour terminer à $19 \%$ d'auteurs publiant seuls en 2007-2016. Cette tendance à la baisse semble avoir été graduelle et indique que les collaborations sont beaucoup plus utilisées aujourd'hui par les auteurs de la revue. Cette baisse se serait actualisée quelque part au tournant des années 2000. Concrètement, cette baisse de l'écriture d'articles en solitaire s'est tra- 
duite par une augmentation de près de $50 \%$ du nombre d'auteurs moyen par article (de 1,3 à 1,9). Chaque auteur avait récemment une médiane de 1,2 coauteur, plus que le double de 1977-1986 (0,5).

TABLEA U 2

Structure des réseaux de coauteurs

\begin{tabular}{|l|c|c|c|c|}
\hline & $1977-1986$ & $1987-1996$ & $\mathbf{1 9 9 7 - 2 0 0 6}$ & $\mathbf{2 0 0 7 - 2 0 1 6}$ \\
\hline Densité & 0,009 & 0,008 & 0,008 & 0,007 \\
\hline Coefficient d'agglomération & 0,803 & 0,684 & 0,901 & 0,615 \\
\hline Degré de centralisation & 0,042 & 0,056 & 0,031 & 0,036 \\
\hline Centralité d'information & 0,009 & 0,009 & 0,012 & 0,017 \\
\hline
\end{tabular}

Le Tableau 2 présente la structure des réseaux de coauteurs de la revue Criminologie. Ces réseaux ne réalisent qu'une infime portion des liens théoriques du réseau. La densité des liens varie en effet de 0,9\% en 1977-1986 à 0,7\% en 2007-2016. Il est difficile de parler d'une diminution dans la densité avec une variation si faible. Le coefficient est cependant extrêmement faible. Bien que la densité des liens soit très limitée, la tendance des auteurs à former des cliques est, elle, importante. Le coefficient d'agglomération est en effet bien supérieur au coefficient de densité et le demeure pour toute la période d'observation. Cette tendance à former des cliques varie dans le temps et son intensité semble monter et descendre en vagues. La formation de cliques serait particulièrement présente pour les années 1997-2006 et au plus creux pour la période suivante, de 2007 à 2016. Ces variations supposent une évolution cyclique de la tendance des réseaux à former des cliques. Évidemment, le petit nombre de périodes nous empêche de faire valoir si ce cycle se répète régulièrement ou s'il s'agit ici d'un artéfact de nos méthodes d'échantillonnage et d'analyse. La capacité des auteurs à rassembler autour d'eux une part plus importante des collaborateurs semblait plus importante dans les 20 premières années de notre étude. La centralisation de degré avait en effet augmenté entre 1977-1986 et 1987-1996, laissant entendre que des auteurs clés avaient émergé. Les deux dernières décennies ont un coefficient similaire $(0,031$ et 0,036$)$ et ce coefficient est près de $50 \%$ de celui de 1987-1996. Cela indique que l'augmentation du nombre d'auteurs pourrait avoir été accompagnée par une diminution de la position de dominance et donc de visibilité de chaque acteur. Même si l'attrait de certains acteurs diminue, les 
acteurs semblent se rapprocher en général avec une médiane de centralité d'information qui augmente constamment (de 0,009 à 0,017). Les collaborations permettent donc aux auteurs de se rapprocher et de former des liens sociaux potentiellement plus propices aux futures collaborations selon Badar et al. (2013).

TABLEA U 3

Recensement des triades

\begin{tabular}{|l|r|r|r|r|}
\hline & \multicolumn{1}{|c|}{$1977-1986$} & \multicolumn{1}{c|}{$1987-1996$} & \multicolumn{1}{c|}{$1997-2006$} & \multicolumn{1}{c|}{$2007-2016$} \\
\hline 0 lien & $273611(97 \%)$ & $486492(98 \%)$ & $1423003(98 \%)$ & $7182095(98 \%)$ \\
\hline 1 lien & $7168(3 \%)$ & $11043(2 \%)$ & $33759(2 \%)$ & $148048(2 \%)$ \\
\hline 2 liens & $35(0 \%)$ & $61(0 \%)$ & $43(0 \%)$ & $627(0 \%)$ \\
\hline 3 liens & $26(0 \%)$ & $44(0 \%)$ & $130(0 \%)$ & $334(0 \%)$ \\
\hline
\end{tabular}

Le recensement des triades présenté dans le Tableau 3 confirme que la densité des liens reste faible durant toute la période d'étude. La quasitotalité des triades sont en effet composées de triades vides $(97 \%$ à $98 \%)$. Les autres types de triades sont cependant en forte augmentation. Les triades à un, deux et trois liens voient leur nombre multiplié par 21, 18 et 13 respectivement durant toute la période de l'étude. Ces augmentations restent cependant plus petites que celles des triades vides qui voient leur nombre multiplié par 26 pendant la même période de temps. La plus forte création de liens semble donc se produire, en ce qui a trait aux dyades, avec des paires d'auteurs, et les cliques d'auteurs qui se forment auront donc tendance à se faire avec des paires d'auteurs plutôt que de larges groupes d'auteurs.

TA B LEA U 4

Structure des réseaux égocentriques de coauteurs

\begin{tabular}{|l|c|c|c|c|}
\hline & 1977-1986 & 1987-1996 & 1997-2006 & 2007-2016 \\
\hline Taille & 1 & 1 & 1 & 2 \\
\hline Densité & $0 \%$ & $0 \%$ & $67 \%$ & $100 \%$ \\
\hline
\end{tabular}

Sur un plan plus local, les collaborations entre auteurs sont aussi limitées. La médiane des coauteurs reste à 1 pendant les trois premières périodes temporelles puis monte à 2 pour la dernière décennie, comme 
le présente le Tableau 4. La densité des liens entre les réseaux égocentriques de coauteurs tend à augmenter cependant, ce qui semble indiquer que les auteurs pourraient puiser dans la banque de relations de leurs propres coauteurs pour trouver de futurs collaborateurs. Le nombre plus élevé d'auteurs par article pourrait aussi expliquer cette augmentation de la densité.

Le Tableau 5 identifie les acteurs qui font partie du cœur de chacune des matrices de relation. Avec les réseaux qui grossissent dans le temps, le cœur est aussi appelé à grandir et il passe de 5 auteurs en 1977-1986 à 23 acteurs en 2007-2016. Nous retrouvons dans cette liste des professeurs bien connus de l'École de criminologie à laquelle la revue Criminologie est rattachée (voir Marc Ouimet, Marie-Marthe Cousineau, Jean-Pierre Guay). D'autres auteurs comme Annie Gendron viennent plutôt du monde de la pratique. Aucun acteur clé n'est présent cependant dans plus d'une période temporelle. L'implication importante dans la revue Criminologie serait donc passagère et possiblement motivée par la nature thématique des numéros de la revue qui fait appel à différentes expertises et à différents auteurs à chaque fois.

TA B LEA U 5

Identification des acteurs au cœur du réseau (par ordre alphabétique)

\begin{tabular}{|c|c|c|c|}
\hline $1977-1986$ & $1987-1996$ & $1997-2006$ & 2007-2016 \\
\hline $\begin{array}{l}\text { amesse-girard_jocelyne } \\
\text { dufour_marie-france } \\
\text { jacques_robert } \\
\text { lacoste_michel } \\
\text { trottier_michel }\end{array}$ & $\begin{array}{l}\text { aubut_jocelyn } \\
\text { mckibben_andre } \\
\text { ouimet_marc } \\
\text { paradis_yves } \\
\text { pellerin_bruno } \\
\text { proulx_jean }\end{array}$ & $\begin{array}{l}\text { ben-datfisher_dahlia } \\
\text { degenna_natacha } \\
\text { grunzeweig_naomi } \\
\text { hodgins_sheilagh } \\
\text { ledingham_jane } \\
\text { schwartzman_alex } \\
\text { serbin_lisa } \\
\text { stack_dale } \\
\text { temcheff_caroline }\end{array}$ & $\begin{array}{l}\text { bourgaultbouthillier_iris } \\
\text { brisebois_rene-andre } \\
\text { bronsard_francine } \\
\text { brunelle_natacha } \\
\text { cousineau_marie-marthe } \\
\text { daspe_marie-eve } \\
\text { desaulniers_valerie } \\
\text { dube_nathalie } \\
\text { dufour_magali } \\
\text { fredette_chantal } \\
\text { gendron_annie } \\
\text { godbout_natacha } \\
\text { guay_jean-pierre } \\
\text { hobbs_julie } \\
\text { lacharite-young_elisabeth } \\
\text { leclerc_danielle } \\
\text { lussier_yvan } \\
\text { mercier_marc-andre } \\
\text { paixao_james } \\
\text { perron_alain } \\
\text { rousseau_michel } \\
\text { sabourin_stephane } \\
\text { tremblay_joel }\end{array}$ \\
\hline
\end{tabular}




\section{Discussion et conclusion}

La revue Criminologie occupe une place importante dans l'histoire de la criminologie francophone. Par son long passé, elle a suivi le développement de la criminologie et les articles qu'elle a publiés permettent de comprendre l'évolution des champs d'intérêt et des approches favorisées par les criminologues. Les autres articles de ce numéro en explorent les détails. Pour sa part, cet article avait pour objectif général de contribuer à l'étude de l'impact de la revue à travers la caractérisation de l'évolution des réseaux de coauteurs de la revue Criminologie au cours des 40 dernières années.

Nos résultats montrent que les auteurs de la revue ont développé leurs réseaux de collaboration au fil du temps, à l'image des chercheurs en général durant la même période. Le nombre d'auteurs participant à la revue a été en forte augmentation, passant de 120 en 1977-1986 à 354 en 2007-2016. Le nombre moyen d'auteurs par article a aussi augmenté de 1,2 à 1,9, entraînant du coup une baisse de la proportion d'auteurs travaillant seuls qui se situe à $19 \%$ pour la dernière période étudiée. Ce mouvement vers le travail collaboratif en sciences est similaire à celui observé dans les recherches passées (Barabâsi et al., 2008; Glänzel et Schubert, 2004; Henriksen, 2016; Uddin et al., 2011; Wuchty et al., 2007). Les proportions exactes des collaborations varient évidemment en fonction des sciences, des jeux de données et de la définition de ce que représente une collaboration (p. ex. : lien de coauteur $v s$ lien de coauteur et remerciement). Toutes les études pointent cependant dans une même direction et la revue Criminologie ne fait pas, à cet égard, bande à part des autres, malgré sa nature de revue francophone en science sociale. Le mouvement vers une plus grande collaboration ne veut pas dire pour autant qu'une seule clique contrôle les publications dans la revue. Au contraire, le fait que les auteurs composant le cœur de la revue changent par décennie et la faible densité laisse supposer que la revue compte sur un corpus d'auteurs varié qui se renouvelle dans le temps.

Plusieurs chercheurs affirment que l'augmentation du travail en équipe découle de l'évolution du travail de chercheur, qui demande toujours plus de ressources financières et humaines et qui impose des contraintes politiques plus sévères (Katz et Martin, 1997; Laudel, 2002; Wuchty et al., 2007). Les recherches deviendraient de plus en plus complexes et nécessiteraient donc que les auteurs collaborent ensemble pour apporter des réponses complexes à des problèmes complexes. Cette 
réalité s'observe dans plusieurs articles publiés dans la revue Criminologie, comme dans celui de Boivin et Lam (2016), qui utilise un sondage d'une grande population, et celui de Descormiers et Corrado (2015), qui est basé sur des dizaines d'entrevues avec des membres de gangs. Cet accent mis sur les recherches d'envergure peut laisser derrière des chercheurs plus qualitatifs dont les recherches plus minutieuses portent sur des objets se prêtant moins à de grandes études. De futurs articles devraient s'interroger sur cette course vers l'avant vers une science toujours plus complexe et les impacts que cette course peut avoir sur les objets d'étude et les connaissances scientifiques générées.

Tout comme dans les études passées sur l'identification d'auteurs clés (Cheong et Corbitt, 2009; Erfanmanesh et al., 2012), la revue Criminologie n'est pas dominée par un seul auteur ni par un petit groupe d'auteurs. Les analyses de cœur/périphérie ont permis de relever un nombre croissant dans le temps d'auteurs importants. Ces auteurs ne sont cependant jamais les mêmes d'une période à l'autre. Notre étude est malheureusement limitée dans sa capacité à analyser les caractéristiques des auteurs faisant partie du cœur du réseau dans chaque décennie. De futures études devraient s'intéresser à ces caractéristiques pour comprendre si la nature francophone et des sciences humaines de la revue Criminologie a un impact sur l'expérience et le profil des auteurs, l'expérience du moins ayant déjà été soulevée comme un facteur important menant à l'acceptation en tant qu'auteur sur une publication (Larivière et al., 2016). Il serait également intéressant de comprendre si des auteurs commencent par publier dans une revue comme Criminologie pour ensuite poursuivre leurs publications dans des revues anglosaxonnes plus populaires auprès d'une communauté scientifique dominée par l'anglais. Cela permettrait ainsi de situer la place de la revue dans l'écosystème scientifique.

La nature thématique de la revue Criminologie pourrait avoir favorisé la rotation des auteurs clés de la revue. Traditionnellement, les éditeurs de chaque numéro de la revue Criminologie étaient libres de sélectionner les auteurs de leur numéro et puisaient donc naturellement dans leur réseau propre de contacts. Comme les auteurs potentiels ne font pas nécessairement partie du cercle de plusieurs éditeurs, il se pourrait fort bien qu'un auteur ne soit invité qu'une seule fois dans sa carrière à participer à un numéro thématique. La durée de l'étude présente aurait pu compenser cette limite, étant donné que des thèmes centraux à la criminologie comme la violence, la victimologie ou encore le lien entre 
les drogues et le crime génèrent assez de recherches pour mériter un numéro thématique, au moins une fois par décennie. Ici encore, le fait que les auteurs clés ne reviennent pas d'une décennie à l'autre indique que la revue mise avant tout sur de nouveaux auteurs, même lorsqu'elle revient sur des thèmes déjà explorés dans le passé, ou que ces auteurs décident de travailler avec de nouveaux partenaires. Cette analyse semble donc indiquer un renouvellement constant des auteurs de la revue, donc un grand dynamisme dans sa direction et son édition.

Parmi ces acteurs clés, il est intéressant de noter que seulement quelques-uns occupent des postes de professeurs à l'École de criminologie de l'Université de Montréal. Longtemps la seule école de formation en criminologie au Québec, l'École compte de ses membres parmi les acteurs clés dans toutes les périodes, sauf celle allant de 1997 à 2006. La revue a donc réussi à sortir du giron très limité de la criminologie à l'Université de Montréal pour puiser dans les connaissances d'auteurs aux horizons plus larges. Il sera intéressant, dans les recherches futures, d'analyser plus finement l'affiliation des auteurs afin de déterminer comment se composent les réseaux locaux des auteurs et si les réseaux sont définis, en tout ou en partie, sur la base des affiliations.

De manière intéressante, l'identification des acteurs clés dans nos matrices donnait des résultats similaires, peu importe la mesure utilisée pour mener à leur détection. Nous avons misé sur la mesure de cœur/ périphérie développée par Borgatti et Everett (2000) et citée dans près de 1500 recherches. D'autres chercheurs avaient plutôt dans le passé utilisé une combinaison de plusieurs mesures de centralité de liens directs, de liens indirects et de proximité, ou encore de nouvelles mesures créées pour les besoins de leur article (Li et al., 2013; Liu et al., 2005; Yan et Ding, 2009). Il nous apparaissait ici plus pertinent de miser sur une méthode établie pour l'identification des acteurs clés. Par ailleurs, combiner plusieurs mesures d'analyses de réseaux est toujours un projet subjectif, car il devient nécessaire de pondérer l'importance des différentes mesures employées. Un tel exercice ne possède que peu d'emprise théorique et laisse toujours le lecteur avec un questionnement sur la fiabilité et la reproductibilité des résultats.

Finalement, bien que la structure des réseaux de coauteurs ait évolué dans le temps, cette transition se fait de manière lente et sans bousculer l'ordre établi. Le recensement des triades nous indique sans surprise que la tendance à former des cliques est présente, mais reste somme toute limitée compte tenu du nombre d'acteurs impliqués. Les mesures d'ana- 
lyses sociométriques restent elles aussi assez limitées dans leur variance. Les augmentations sont notables en termes de pourcentage, mais restent dans l'absolu beaucoup plus modérées. Cette stabilité qui va quelque peu à l'encontre des mouvements dans les milieux plus anglo-saxons laisse supposer que la criminologie francophone évolue à son rythme et quelque peu en marge des réseaux plus traditionnels de recherche. Des recherches futures devraient s'intéresser à l'impact de cette stabilité sur la performance des auteurs, que ce soit ce qui a trait aux citations reçues ou encore au nombre d'articles écrits. Une plus grande place de collaborations augmente en effet le nombre d'articles publiés et permet ainsi de décupler le nombre de publications réalisées. Les collaborations permettent aussi de partager l'existence des publications afin qu'elles soient plus présentes dans la tête des chercheurs au moment d'écrire leurs articles.

Cet article se voulait une première analyse francophone d'un réseau d'auteurs lui aussi, francophone. Bien que la période étudiée soit importante (40 ans), les recherches futures devraient s'intéresser au monde francophone à travers d'autres revues autant en criminologie que dans d'autres sciences. Il est en effet fort probable que les réseaux d'auteurs des revues des sciences pures et de la nature aient des structures qui se différencient de celles des revues en sciences humaines. Les futures recherches devraient aussi s'intéresser davantage au concept de performance qui est au cœur des recherches dans le monde anglo-saxon. Les chiffres sur les citations de chaque article ne sont pas évidents à obtenir et peuvent être remis en question, mais une étude du lien entre la structure des liens et la performance serait de mise dans le monde francophone. Étant donné son public plus limité, il sera intéressant de comprendre si la performance est tout aussi liée à la structure des réseaux. Cette performance pourrait aussi s'appliquer aux revues ellesmêmes afin de chercher à comprendre la visibilité des revues (à travers leurs citations reçues) et la structure de leurs réseaux d'auteurs. Ce travail permettrait de mieux positionner les revues les unes par rapport aux autres et favoriser la diffusion de mesures et stratégies utilisées par les meilleures revues pour se faire connaître.

\section{Références}

Abbasi, A., Altmann, J. et Hossain, L. (2011). Identifying the effects of coauthorship networks on the performance of scholars: A correlation and regression analysis of performance measures and social network analysis measures. Journal of Informetrics, 5(4), 594-607. 
Abbasi, A., Chung, K. S. K. et Hossain, L. (2012). Egocentric analysis of coauthorship network structure, position and performance. Information Processing $\mathfrak{F}$ Management, 48(4), 671-679.

Acedo, F. J., Barroso, C., Casanueva, C. et Galán, J. L. (2006). Co-authorship in management and organizational studies: An empirical and network analysis. Journal of Management Studies, 43(5), 957-983.

Babchuk, N., Keith, B. et Peters, G. (1999). Collaboration in sociology and other scientific disciplines: A comparative trend analysis of scholarship in the social, physical, and mathematical sciences. The American Sociologist, 30(3), 5-21.

Badar, K., Hite, J. M. et Badir, Y. F. (2013). Examining the relationship of coauthorship network centrality and gender on academic research performance: The case of chemistry researchers in Pakistan. Scientometrics, 94(2), 755-775.

Barabâsi, A. L., Jeong, H., Néda, Z., Ravasz, E., Schubert, A. et Vicsek, T. (2008). Evolution of the social network of scientific collaborations. Physica A : Statistical Mechanics and its Applications, 311(3), 590-614.

Boivin, R. et Lam, R. (2016). Sondages Web, opinion publique et performance policière. Criminologie, 49(1), 225-245.

Borgatti, S. P. et Everett, M. G. (2000). Models of core/periphery structures. Social Networks, 21(4), 375-395.

Borgatti, S. P., Everett, M. G. et Freeman, L. C. (2002). Ucinet 6 for Windows: Software for social network analysis. Harvard, MA: Analytic Technologies.

Börner, K., Dall'Asta, L., Ke, W. et Vespignani, A. (2005). Studying the emerging global brain: Analyzing and visualizing the impact of co-authorship teams. Complexity, 10(4), 57-67.

Cheong, F. et Corbitt, B. J. (2009). A social network analysis of the co-authorship network of the Pacific Asia Conference on information systems from 1993 to 2008. Proceedings of the Pacific Asia Conference on Information Systems, 23.

Chung, K. S. K. et Hossain, L. (2009). Measuring performance of knowledgeintensive workgroups through social networks. Project Management Journal, $40(2), 34-58$.

Clarke, B. L. (1964). Multiple authorship trends in scientific papers. Science, 143(3608), 822-824.

De Stefano, D., Fuccella, V., Vitale, M. P. et Zaccarin, S. (2013). The use of different data sources in the analysis of co-authorship networks and scientific performance. Social Networks, 35(3), 370-381.

Descormiers, K. et Corrado, R. (2015). Le processus de désaffiliation aux gangs. Le rôle des facteurs individuels et collectifs. Criminologie, 48(2), 139-163.

Erfanmanesh, M., Rohani, V. A. et Abrizah, A. (2012). Co-authorship network of scientometrics research collaboration. Malaysian Journal of Library $\mathfrak{F}$ Information Science, 17(3), 73-93.

Glänzel, W. (2002). Coauthorship patterns and trends in the sciences (19801998): A bibliometric study with implications for database indexing and search strategies. Repéré à https://www.ideals.illinois.edu/bitstream/ handle/2142/8409/librarytrendsv50i3k_opt.pdf? seq. 
Glänzel, W. et Schubert, A. (2004). Analysing scientific networks through co-authorship. Dans H. F. Moed, W. Glänzel et U. Schmoch (dir.), Handbook of quantitative science and technology research (p. 257-279). New York, NY: Springer.

Gossart, C. et Özman, M. (2008). Co-authorship networks in social sciences: The case of Turkey. Scientometrics, 78(2), 323-345.

Henriksen, D. (2016). The rise in co-authorship in the social sciences (19802013). Scientometrics, 107(2), 455-476.

Katz, J. S. et Martin, B. R. (1997). What is research collaboration? Researcb Policy, 26(1), 1-18.

Kronegger, L., Ferligoj, A. et Doreian, P. (2011). On the dynamics of national scientific systems. Quality \& Quantity, 45(5), 989-1015.

Kuhn, T. (1981). What are scientific revolutions. Boston, MA: MIT.

Largeron-Leteno, C. et Massard, N. (2001). La géographie des collaborations scientifiques en France: une étude de la structuration des co-publications entre départements. Revue d'économie régionale et urbaine, (1), 39-52.

Larivière, V., Desrochers, N., Macaluso, B., Mongeon, P., Paul-Hus, A. et Sugimoto, C. R. (2016). Contributorship and division of labor in knowledge production. Social Studies of Science, 46(3), 417-435.

Laudel, G. (2002). What do we measure by co-authorships? Research Evaluation, 11(1), 3-15.

Lee, S. et Bozeman, B. (2005). The impact of research collaboration on scientific productivity. Social Studies of Science, 35(5), 673-702.

Li, E. Y., Liao, C. H. et Yen, H. R. (2013). Co-authorship networks and research impact: A social capital perspective. Research Policy, 42(9), 1515-1530.

Liu, X., Bollen, J., Nelson, M. L. et Van De Sompel, H. (2005). Co-authorship networks in the digital library research community. Information Processing $\sim$ Management, 41(6), 1462-1480.

Lu, H. et Feng, Y. (2009). A measure of authors' centrality in co-authorship networks based on the distribution of collaborative relationships. Scientometrics, 81(2), 499-511.

Melin, G. et Persson, O. (1996). Studying research collaboration using coauthorships. Scientometrics, 36(3), 363-377.

Morel, C. M., Serruya, S. J., Penna, G. O. et Guimarães, R. (2009). Co-authorship network analysis: A powerful tool for strategic planning of research, development and capacity building programs on neglected diseases. Public Library of Science, 3(8), e501.

Newman, M. E. (2004). Coauthorship networks and patterns of scientific collaboration. Proceedings of the National Academy of Sciences, 101(1), 52005205.

Paul-Hus, A., Mongeon, P., Sainte-Marie, M. et Larivière, V. (2017). The sum of it all: Revealing collaboration patterns by combining authorship and acknowledgements. Journal of Informetrics, 11(1), 80-87.

Pavlov, M. et Ichise, R. (2007). Finding experts by link prediction in coauthorship networks. Proceedings of the 2nd international conference on finding experts on the web with semantics. Busan, Corée du Sud. 
Perretti, F. et Negro, G. (2006). Filling empty seats: How status and organizational hierarchies affect exploration versus exploitation in team design. Academy of Management Journal, 49(4), 759-777.

Popper, K. (2014). Conjectures and refutations: The growth of scientific knowledge. New York, NY: Routledge.

Reichers, A. E. (1987). An interactionist perspective on newcomer socialization rates. Academy of Management Review, 12(2), 278-287.

Taylor, A. et Greve, H. R. (2006). Superman or the fantastic four? Knowledge combination and experience in innovative teams. Academy of Management Journal, 49(4), 723-740.

Uddin, S., Hossain, L., Abbasi, A. et Rasmussen, K. (2012). Trend and efficiency analysis of co-authorship network. Scientometrics, 90(2), 687-699.

Velden, T., Haque, A. U. et Lagoze, C. (2010). A new approach to analyzing patterns of collaboration in co-authorship networks: Mesoscopic analysis and interpretation. Scientometrics, 85(1), 219-242.

Wuchty, S., Jones, B. et Uzzi, B. (2007). The increasing dominance of teams in production of knowledge. Science, 316(5827), 1036-1041.

Yan, E. et Ding, Y. (2009). Applying centrality measures to impact analysis: A coauthorship network analysis. Journal of the Association for Information Science and Technology, 60(10), 2107-2118.

Yin, L. C., Kretschmer, H., Hanneman, R. A. et Liu, Z. Y. (2006). Connection and stratification in research collaboration: An analysis of the COLLNET network. Information Processing $\mathcal{O}$ Management, 42(6), 1599-1613.

Yu, Q. Shao, H. et Duan, Z. (2011). Research groups of oncology co-authorship network in China. Scientometrics, 89(2), 553-567.

\section{Co-authorship networks: 40 years of collaborations in the journal Criminologie}

ABSTRACT - While scientific research usually seeks to understand the world around it, it occasionally looks at itself in an attempt to better understand how scientific knowledge is produced. These attempts have resulted in an important literature on the networks of collaborations that develop between the authors of scientific texts. The general objective of such research is to explain the structure of collaborations and to identify the corollaries such collaborations have in the academic world. This article aims to fill a gap in knowledge about francophone collaboration networks by studying 40 years of collaborations between Criminologie authors. The methodological approach is both egocentric and sociometric, which makes it possible to capture the links between co-authors. Results show an increase in collaborations over time but not necessarily the creation of a single community of authors who control the diffusion of knowledge in the journal. While there are key players, they change over time.

KEYWORDS - Co-authorship networks; social network analysis; longitudinal research; francophone networks. 


\section{Las redes de coautores: 40 años de colaboraciones en la revista Criminologie}

RESUMEN - Generalmente, la investigación científica busca entender la naturaleza del conocimiento científico, pero, eventualmente, se enfoca también hacia sí misma para poder comprender mejor su génesis. Esta búsqueda se ha traducido en una literatura importante sobre las redes de colaboraciones entre autores para la producción de textos científicos. El objetivo general de este campo de investigación es explicar la estructura de las colaboraciones, y determinar los corolarios del rendimiento en el mundo universitario. El presente artículo intenta desarrollar el conocimiento sobre las redes de colaboración francófonas, estudiando 40 años de colaboraciones entre autores de la revista Criminologie. La metodología adopta una aproximación egocéntrica y sociométrica, y caracteriza los vínculos entre los coautores de la revista. Los resultados demuestran un aumento de las colaboraciones a través del tiempo, pero sin que, necesariamente, se cree una sola y única comunidad de autores que controle la difusión del conocimiento en la revista. Los actores clave son presentados, pero no demuestran ninguna estabilidad en el tiempo.

PALABRAS CLAVE - Redes de coautores, análisis de redes, estudios longitudinales, redes francófonas. 